\title{
A DYNAMIC SPECTRUM ACCESS OPTIMIZATION MODEL FOR COGNITIVE RADIO WIRELESS SENSOR NETWORK
}

\author{
Saroja T.V ${ }^{1}$, Lata L. Ragha ${ }^{2}$ and Satyendra Kumar Sharma ${ }^{3}$ \\ ${ }^{I}$ Department of Computer Engineering, Pacific Academy of Higher Education and Research University, India \\ ${ }^{2}$ Department of Computer Engineering, Fr. Conceicao Rodrigues Institute of Technology, India \\ ${ }^{3}$ Modern Institute of Technology and Research Centre, India
}

\begin{abstract}
The availability of low cost and tiny sensor devices have resulted in increased adoption of wireless sensor network (WSN) in various industries and organization. The WSN is expected to play a significant role in future internet based application services. WSN has been adopted in healthcare, disaster management, environment monitoring and so on. The low-cost availability of smart devices has led to increased use of wireless devices such as Bluetooth, Wi-Fi etc. Therefore, cognitive radio network plays a significant role in handling spectrum efficiently. The emerging internet access technology such as $4 G$ and $5 G$ network which is expected to come in near future is going to make cognitive spectrum access more challenging. The existing cognitive radio based WSN is not efficient in utilizing spectrum. They induce high collision due to interference and improper channel state information. To address, this work present an efficient distributed opportunistic spectrum access for wireless sensor network. The channel availability of likelihood distribution is computed using continuous-time Markov chain considering primary transmitting users temporal channel usage channel pattern and spatial distribution. The simulation outcome shows the proposed model achieves significant performance improvement over existing model. The proposed model improves the overall spectrum efficiency of cognitive radio wireless sensor network in terms of throughput, packet transmission and collision.
\end{abstract}

\section{Keywords:}

Cognitive Radio Network, Wireless Sensor Network, MAC, Spectrum

\section{INTRODUCTION}

A WSN is self-composed of low cost and tiny sensor devices which are deployed in remotely dense area. The sensor devices are used to sense wide variety of conditions such as tracking object, pressure, temperature etc. [1]. The next generation wireless sensor networks need to consider the integration of internet of things (IoT) [2] where the sensors are connected to internet dynamically for various applications [3- 8] such as multimedia, IoT and big data, E-commerce etc. The multimedia based application service requires high data rate, low energy depletion and more spectrum availability [9], [10], and [11]. However the $2.4 \mathrm{GHz}$ radio frequency band is very crowded due to the availability of wireless technologies such as Wi-Fi, Bluetooth technology and the 5G network is expected to come in near future which requires efficient spectrum access mechanism. As a result cognitive network plays an important role in solving spectrum scarcity problems and aid in providing efficient spectrum access for future wireless technology and its application. Specifically for resource constrained wireless sensor networks. The architecture of cognitive radio wireless sensor network is shown in Fig.1.

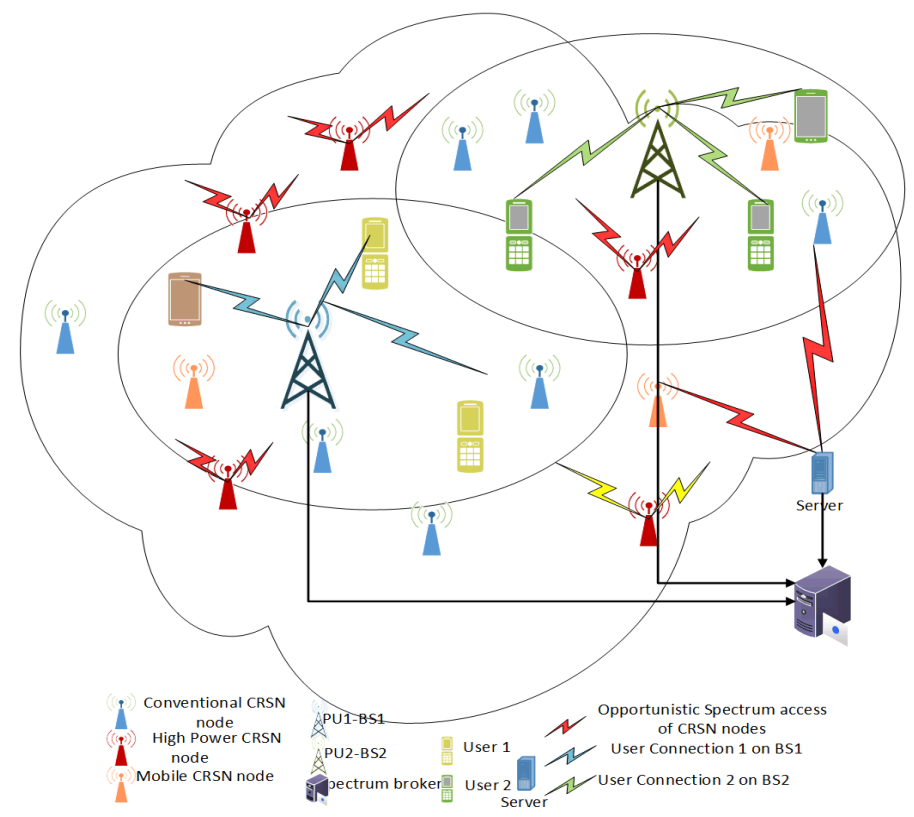

Fig.1. Architecture of Cognitive Radio Wireless Sensor Network

To develop an efficient spectrum access in cognitive radio wireless network it needs to further consider issues and challenges such as:

Opportunistic channel usage for bursty traffic: Once an event is detected in the WSN, packet burst is generated by the sensor nodes. At this time, densely deployed sensor nodes also try to acquire the channel in event area which may lead to the packet collision and it reduces the overall network performance, resulting in communication overhead, delay etc. Here, sensor nodes with cognitive radio ability opportunistically access to multiple substitute channels to reduce these major challenges.

Dynamic spectrum access: Generally fixed spectrum allocation scheme is used in the WSN deployment. WSN can be operated on unlicensed and licensed band. Spectrum are directly associated with the cost which increase the network cost. However WLAN can also the unlicensed band. Therefore, WSNs experience the overloaded spectrum issue [12]. To increase the network performance, opportunistic spectrum sensing techniques must be used in WSN as well.

Using adaptability to reduce power consumption: Power consumption in the WSN is also a key issue. Due to loss of packet and retransmission of same packet more energy is consumed in the network. If nodes are capable to use Cognitive radio it would be able to adapt the channel condition. In this way loss, free transmission is increased which reduces the energy consumption. 
Overlaid deployment of multiple concurrent WSN: As increased in usages of WSNs, a particular area can host various networks, which require the management of spectrum at real time or dynamic management that work well for partially overlapped sensor network, and maintain the system performance in terms of communication.

Access to multiple channels to conform to different spectrum regulations: As we know that availability of the spectrum frequency is different for all the countries. A band which is available in India may not be available in some other country. So, if sensor nodes are designed with preset frequency band it might create a problem for the user. This problem can be overcome by using cognitive radio capability; which changes their communication frequency based on the availability of spectrum.

Effective mechanisms are needed for spectrum access by sensor device to achieve throughput requirement of multimedia service [13], [14] and [15]. The wireless environment is getting more complicated and it is getting more problematic in obtaining wireless network information for cognitive user. The $5 \mathrm{G}$ network architecture is expected to provide ultra-low power and superefficient spectrum access to support large density of users with less delay and limited bandwidth which will be a challenging task for existing crowded spectrum. Therefore, distributed channel access considering unknown network information is been the existing research trends of cognitive radio wireless sensor network.

There has been various existing work [16] [17] that have studied the problem of distributed spectrum access considering unknown network information considering single cognitive user. In [18] the authors presented multiuser based spectrum access model. When multi users are presented in network it induces Collison. To address this in [18] the presented an adaptive random access model and in [19] authors presented fair access model and [20] authors presented priority access model to reduce the collision among cognitive users. It is seen from exiting research [21] [22] that most of these schemes are limited to provide only one channel at a time to a cognitive user. Thus, if a selected channel is busy the user needs to wait for another slot. However if another channel is free at that time the spectrum is wasted.

To solve spectrum resource scarcity and utilize bandwidth more efficiently and improve the throughput of IOT based application under multi user and multichannel environment, [23] presented Multi-Armed Bandit Channel Access Scheme. They presented a distributed learning scheme to improve usage of idle spectrum. However, adopting Multi-Armed Bandit Channel Access Scheme may fail to find more spectrum opportunities for larger network [24] and mobility of secondary user is not taken into consideration.

To address the research issues, firstly we analyze the channel availability of CR-WSN considering the mobility of sensor node (secondary user). Then, by exploiting the temporal and spatial channel usage characteristic of primary user a distributed opportunistic channel access mechanism for multi user and multichannel CR-WSN is presented. The likelihood distribution of availability of channel is computed using continuous-time Markov chain. Simulation outcomes shows the efficiency of proposed model over existing approaches [23] in terms of throughput, collision and successful transmission.

The contribution of research work are as follows,
- This work firstly presented a mechanism to compute the likelihood of channel availability considering mobile secondary nodes.

- Secondly, presented a distributed opportunistic spectrum access mechanism for multi user and multi-channel CRWSN.

- The proposed model minimize collision and maximize network throughput.

The paper is organized as follows: The proposed CR-WSN model is presented in section two. In section three the simulation outcome and analysis is presented. The last section the paper is concluded with future research.

\section{LITERATURE SURVEY}

Adoption of cognitive radio for WSN has gained huge attention in recent time. However there exists several challenges in designing efficient cognitive radio WSN for spectrum access of unused band of licensed primary user. Here we survey some of existing methodology that has been developed in recent time for cognitive radio WSN.

In [25] the authors adopted cognitive radio based wireless sensor architecture. They presented dynamic spectrum access of unused band of licensed user of cognitive radio. The sensor device uses these bands for communication with its neighbor or sink. They identified the challenges that existed in spectrum access of resource constrained WSN and proliferation of services and wireless devices due to scarcity. Therefore it is important to satisfy the needs of WSN and cognitive radio in order to utilize the benefits of cognisions in WSN. To achieve this, they presented an energy efficient WSN for multi-channel MAC. They developed an asynchronous duty cycle for spectrum access to satisfy both cognitive radio and WSN. The simulation is conducted and evaluated with Multi-channel MAC show performance improvement in terms of throughput and delay. The drawback of this model is that their model performs well for large network but for smaller network it degrades which shows that their model is no robust and dynamic.

In [26] the authors presented a cooperative transmission by adopting MIMO (Multiple input Multiple output) for large scale wireless sensor network. Here the cognitive radio is adopted for transmission of uplink in wireless sensor network. Their model identified the issues of network capacity for opportunistic spectrum sharing, interference caused due to spectrum sharing and energy degradation of sensor device in the presence of primary user. To address these issues, they presented cluster based cooperative sensor network. They adopted OFDM (orthogonal frequency division multiplexing) for cooperative transmission and used PSO (particle swarm optimization) for cluster selection. Their outcome showed better convergence than existing search model and the drawback of this model is that their model is not distributed thus it is not suitable for larger network.

In [27] the authors surveyed various existing cognitive radio based wireless sensor networks. They identified that it is important to develop a strategy for intelligent allocation of radio resource to perform efficient and dynamic spectrum access policy. When developing a cognitive radio for WSN, the model needs to consider the resource and energy constraint of WSN. The radio 
allocation schemes are broadly classified into cluster based, distributed and centralized. The performance benefits of strategy depend on criteria such as throughput maximization, energy efficiency, fairness, assurance of QoS and so on. The overall survey shows that considerable work has been carried out and limited research is carried out for cluster and distributed approach. The problem with distributed spectrum access mechanism is that it requires efficient optimization technique to detect the channel condition.

In [28] they identified that in cognitive radio network, to detect unused channel it needs either predefined sensing sequence or the random channel access. Due to this it is difficult in achieving efficiency in resource constrained sensor network. To address this they presented reinforcement learning for channel sensing in cluster based cooperative wireless sensor network. The utility policy is defined by adopting Markov Decision problem to minimize sensing. The outcome shows improvement of detection of primary user and sensing energy cost over existing greedy search approach.

In [29] the authors addressed the energy efficiency issues of sensor network when incorporating cognitive radio. The channel sensing of cognitive radio will induce energy overhead head for resource constrained sensor device. To address this efficient spectrum sensing strategy needs to be developed. They presented spectrum sensing time period model to predict the current channel information status for secondary user channel sensing. They adopted a Markov chain model for dynamic spectrum sensing which adopt listen before talk strategy. The simulation is conducted for varying interval time. The outcome showed that Markov chain model improves energy efficiency of cognitive radio based WSN but they considered varying network density.

In [30] they presented a real time monitoring of carbon dioxide by adopting cognitive radio wireless ad-hoc network. The experiments are conducted for different indoor environment considering Carrier sense multiple access with collision avoidance as the radio model. The results are presented in terms of end-to-end delay and throughput for both normal and cognitive environment. The outcome shows adopting cognitive radio for wireless sensor network improves the network performance in terms of throughput and network end-to-end delay. However, it induces spectrum wastages. To address, in [23] presented a MultiArmed Bandit Channel Access Scheme to minimize bandwidth wastage for multi user and multi-channel CR-WSN. They presented a distributed learning scheme to improve usage of idle spectrum. However, their scheme may fail to find more spectrum opportunities for larger network and mobility of secondary user is not taken into consideration.

Extensive research carried outcome shows that the cognitive radio plays significant part in improving the overall network performance of wireless sensor network. It is important to utilize bandwidth efficiently in order to provision IoT based application on multi user and multi-channel CR-WSN. The Markov chain model improves the prediction accuracy of channel state information for efficient spectrum sensing. The overall survey shows that very limited work is carried out in distributed spectrum sensing for multi user and multichannel cognitive radio wireless sensor network. There is a need to develop a better optimization technique to improve accuracy for channel state prediction for efficient channel access. In next section, the proposed channel state and distributed opportunistic spectrum access optimization by using continuous-time Markov chain for CR-WSN is presented.

\section{PROPOSED OPPORTUNISTIC SPECTRUM ACCESS TECHNIQUE FOR CR-WSN}

Let us consider a cognitive radio wireless sensor network, consisting of a set of primary user such as the base stationlsink and the unlicensed secondary user (sensor devices). A sensor device is fitted with cognitive radio for opportunistic access of licensed spectrum. Let $H$ be the set of nonempty opportunistic channel that can be accessed by sensor devices. There exists limited channel availability for sensor device due to characteristics nature of primary user and sensor devices positions/locations. The characteristic of spectrum opportunity depends on the channel availability to sensor device which is defined as the amount of time the channel is available to sensor devices or not. The accessibility of channel $c, c \in H$, for a sensor device is defined by the temporal channel usage pattern of primary user and spatial distribution on channel $\mathrm{c}$ and the location of sensor device.

Therefore estimating the channel availability plays an important role in improving the QoS of secondary user and improves utilization of spectrum. Let us consider a continuoustime Markov chain that considers primary user channel usage pattern and the secondary user location. The continuous-time Markov chain consists of three modes $M_{I}, M_{B}$ and $M_{Z} . M_{I}$ is the mode in which a node is within coverage of idle primary transmitting user that transmit on channel $c, M_{B}$ is the mode where in a node is within coverage of active primary transmitting user that transmit on channel $c$ and $M_{Z}$ is the mode where in a node is outside the coverage area of primary transmitting user that transmit on channel $c$. When the position of sensor devices is changed the mode changes from one form to another form and channel is inaccessible only when mode is in $M_{B}$. The mode $M_{I}$ and $M_{Z}$ can be merged to one mode where the channel is accessible to the sensor device which is represented by $M_{X}$. The mode of unavailability of channel is represented by $M_{N}$.

Let $K_{X, c}$ and $K_{N, c}$ be the time duration of sensor node in $M_{X}$ and $M_{N}$ respectively. The likelihood distribution of channel availability of $K_{X, c}$ and $K_{N, c}$ is important to compute the transition mode of $M_{I}$, $M_{B}$ and $M_{Z}$ i.e. Let $K_{, c}$ be the time period of a sensor device when it is in range of primary transmitting user on channel $c$ and $K_{{ }^{\prime}, c}$ be the time period when a sensor device is outside the range of primary transmitting user on channel $c$. Thus the rate of transition modes depend on the likelihood distribution of $K_{, c}$ and $K_{,, c}$. To compute $K_{, c}$ and $K_{, c}$ a two-dimensional Markov chain model is considered.

The spatial distribution of Primary transmitting user that process on common channel $\mathrm{c}$ are distributed in square area. The distance among two adjacent primary transmitting users in vertical direction is represented as $A_{q, c}$ and a horizontal direction is represented as $A_{p, c}$. The square area of $2 T_{c}$ is considered for coverage area of Primary transmitting user where $T_{c}<\min \left(A_{p, c}, A_{q, c}\right)$ to avoid overlapping section among primary transmitter user. To protect the primary transmission, we consider that approximate coverage area greater than the actual coverage area. 
The temporal usage of pattern of primary transmitting user that communicates on channel $c$ is exhibited as idle (primary transmitting user does not communicate) and busy (primary transmitting user is active and communicate) mode. The sensor nodes in the coverage area of primary transmitting user do not have access to transmit on same channel during the transmission period of primary transmitting user in order to avoid the interference with primary user network. The periodic length of idle/busy mode is exhibited as an exponential random variable as $\mu_{I} / \mu_{B}$, that is,

$$
K_{I, c} \sim \mathrm{e}\left(\mu_{I, c}\right) \text { and } K_{B, c} \sim e\left(\mu_{B, c}\right)
$$

where, $F \sim e(\mu)$ indicates that variable $F$ is an exponential distribution with $\mu$.

$$
\alpha_{I, c}=\frac{\mu_{B, c}}{\mu_{I, c}}+\mu_{B, c}
$$

are the steady mode likelihood that primary transmitter being active on channel $c$ and

$$
\alpha_{B, c}=1-\alpha_{I, c}
$$

are the steady mode likelihood that primary transmitter being inactive on channel $c$.

Therefore the mean fraction channel availability of an area at any instance of channel $c$ is represented by $\omega_{c}$.

$$
\omega_{c}=\left(1-\beta_{c}\right)+\beta_{c} \alpha_{I, c}=1-\beta_{c} \alpha_{B, c}
$$

where, $\beta_{c}=\frac{4 T^{2}}{A_{l, i}^{2}}$.

The sensor nodes are considered to be in range of primary transmitting user $\varphi_{T}$ to optimize $K$. . Let $\varphi_{T}$ be square of length $V_{T, c}$. For easiness, let $A_{S}=A_{P}=A$ and $A_{L P, c}=A_{L S, c}=A_{L, c}$. The length of all is normalized by $A$, i.e. $V_{T, c}=2 T_{c} / A$. To obtain $K$, a 2-D Markov chain is used. Let each location $(g, u)$ within $\varphi_{T}$ be in mode Z_ $(\mathrm{g}, \mathrm{u})$. The Markov chain of all these modes as:

$$
Z_{g, u}=\left\{Z_{1,1}, Z_{1,2}, \ldots, Z_{1, V_{T, C}}, Z_{2,1}, Z_{2,2}, \ldots, Z_{V_{T, c}, V_{T, c}}\right\}
$$

It is considered that sensor nodes are in mode $Z_{g, u}$ if it changes its position from location $(g, u)$ to its adjacent location $(g, u)$, the mode transition also takes place from $(g, u)$ to $(g, u)$. The sensor devices that are in the boundary area of primary transmitting user are considered as diminishing modes. Let $B_{X}$ be the collection of diminishing modes sets. Let $W$ be transition number of sensor device leaving area $\varphi_{T}$ that is the mode of transition in mode $B_{X}$. The approximation of $K$ is done by $W \times \delta_{k}$, where $\delta_{k}$ is period that a sensor node in network location. The likelihood distribution of $W$ needs to be computed to compute the likelihood distribution of $K$. The transition likelihood of $Z_{g, u}$ belonging to $B_{X}$ is obtained as follows:

$$
\left\{\begin{array}{c}
L\left(Z_{g, u} \mid Z_{g, u}\right)=1 \\
L\left(O \mid Z_{g, u}\right)=0
\end{array}\right.
$$

The transition likelihood of $Z_{g, u}$ that does not belong to $B_{X}$ is obtained as follows

$$
\left\{\begin{array}{c}
L\left(Z_{g, u-1} \mid Z_{g, u}\right)=L_{a} \\
L\left(Z_{g, u+1} \mid Z_{g, u}\right)=L_{r} \\
L\left(Z_{g-1,1} \mid Z_{g, u}\right)=L_{n} \\
L\left(Z_{g+1,1} \mid Z_{g, u}\right)=L_{j} \\
L\left(O \mid Z_{g, u}\right)=0
\end{array}\right.
$$

The matrix $L$ is sparse and the likelihood distribution of modes after $w$ transition is represented by $\tau^{w}$. The initial mode likelihood distribution is represented by $\tau^{0}$. It holds that $\tau^{w}=$ by $\tau^{0} L^{w}$. It is conceivable that nodes to be in any modes in $\varphi_{T}$ at the beginning of time $k_{0}$, excluding devices in $B_{X}$. Let the set of probable modes be $B_{C}$. The cardinality of $B_{C}$ is represented by, $Z_{C}$, which is computed as $Z_{C}=\left|B_{C}\right|=\left(V_{T, c}-2\right)^{2}$. Rest of the initial probable path is considered to have equivalent likelihood, therefore $\tau^{0}$ is computed as follows:

$$
\tau_{(g, u)}^{(0)}\left\{\begin{array}{cc}
l C=\frac{1}{Z_{C}}= & \frac{1}{\left(V_{T, c}-2\right)^{2}} Z_{g, u}=0 \\
0 & \text { else }
\end{array}\right.
$$

where, $l C$ is the likelihood of every probable initial modes. The likelihood of $W$ is not greater than $w$ is as follows,

$$
L(W>w)=\sum_{Z_{g, u} \in B_{X}} \tau^{(w)} .
$$

Thus the likelihood density function of $W$ is as follows:

$$
\begin{gathered}
L(W=w)=L(W \leq w)-L(W \leq w-1) \\
=\sum_{z_{g, u} \in B_{X}} \tau^{(w)}-\sum_{Z_{g, u} \in B_{X}} \tau^{(w-1)}
\end{gathered}
$$

Therefore, if all the modes in $B_{X}$ is considered as $M_{\text {" }}$ and other mode to $M_{\cdot}$, the $Z_{g, u}$ is considered to be 2-D i.e. two modes Markov chain $\left\{M_{.}, M_{n}\right\}$. The sensor devices is initially in $M_{\text {.. In }}$ every transition the sensor devices will remain in $M$, with

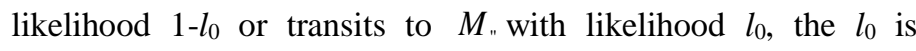
computed as

$$
l_{0}=\frac{1}{Z_{C}} \sum_{Z_{g, u} \in B_{X}} \sum_{Z_{g, u} \in B_{X}} L\left(Z_{g, u} \mid Z_{g, u}\right)
$$

The sensor devices don't change its location till the mode transits to $M_{n}$. Therefore, the transition number of sensor devices before leaving the primary transmitting user area is said to follow a geometric distribution with $1=l_{0}$. Therefore $K$, is computed by an exponential distribution.

To optimize $K_{\text {n }}$, let us consider a square area $\varphi_{T}$ which is torus and length of network $V_{Q, c}=P_{L, c} / P$ ]. Here the $\varphi_{T}$ are considered as diminishing mode. The transition matrix $L$ is computed similarly as Eq.(4) and Eq.(5). 


$$
\left\{\begin{array}{l}
L\left(Z_{g, V_{Q}} \mid Z_{g, 1}\right)=L_{p} \\
L\left(Z_{g, 1} \mid Z_{g, V_{Q}}\right)=L_{k} \\
L\left(Z_{V_{Q}, u} \mid Z_{1, u}\right)=L_{n} \\
L\left(Z_{1, u} \mid Z_{V_{Q}, u}\right)=L_{q}
\end{array}\right.
$$

Similar to $K$, the probable initial mode are considered to be equal with the likelihood. The collection of probable initial mode set is represented by $\left|B_{C}\right|=V_{J, c}^{2}-V_{R, c}^{2}$. Therefore the initial state likelihood distribution $\tau^{(0)}$ is computed as follows:

$$
\tau_{(g, u)}^{(0)}\left\{\begin{array}{cc}
l C=\frac{1}{V_{J, c}^{2}-V_{R, c}^{2}} & Z_{g, u} \in B_{C} \\
0 & \text { else }
\end{array}\right.
$$

Similar to Eq.(8) the likelihood mass of $\bar{W}$ is computed as follows:

$$
\begin{gathered}
L(\bar{W}-w)=L(\bar{W} \leq w)-L(\bar{W} \leq w-1) \\
=\sum_{z_{g, u} \in B_{X}} \tau^{(w)}-\sum_{z_{g, u} \in B_{X}} \tau^{(w-1)}
\end{gathered}
$$

where, $\bar{W}$ represent the transition number before a sensor devices changes its location to primary transmitting user area of coverage. The experimental and simulation analysis is performed in next chapter below.

\section{SIMULATION RESULT AND ANALYSIS}

The system environment used is windows 7, 64-bit quad core operating system with $12 \mathrm{~GB}$ of ram. The authors have used dot net framework 4.0 and C\# 6.0 programming language for the proposed and existing work [23] and conducted experimental study on following parameters, throughput achieved per channel, successful packet transmission and packet collision per channel. The network area is fixed to $30 \times 30$ meters, the sensor nodes considered are 25 and 50 devices, and the spectrum consists of 8 timeslots and 3 frequency slots. For every second, 40 bytes of message is generated per sensor node and random selection of source and destination sensor devices is considered. The BPSK modulation scheme is used which support maximum bandwidth of $3 \mathrm{Mbps}$.

In Fig.2, the simulation outcome of packet collision is shown. The sensor nodes are varied from 25 and 50 sensor devices and outcome shows that the proposed model reduces packet collision over existing model. A collision reduction of $22.37 \%$ is achieved when sensor nodes are equal to 25 and collision reduction of $8.79 \%$ is achieved when sensor nodes are equal to 50. It is seen from graph that as we increase the number of sensor devices the collision also increases for both proposed and existing approach.

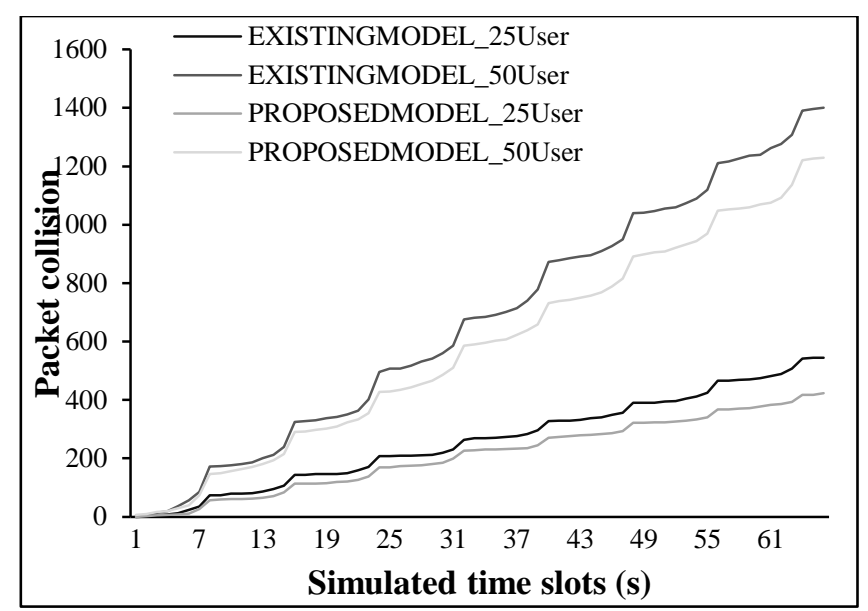

Fig.2. Packet collision for varied sensor devices

In Fig.3, the simulation outcome of throughput achieved per channel is shown. The sensor nodes are varied from 25 and 50 sensor devices and outcome shows that the proposed model improves throughput over existing model. An average throughput improvement of $33.21 \%$ is achieved when sensor node is equal to 25 and throughput improvement of $27.38 \%$ is achieved when sensor node is equal to 50 which are shown in Fig.4.

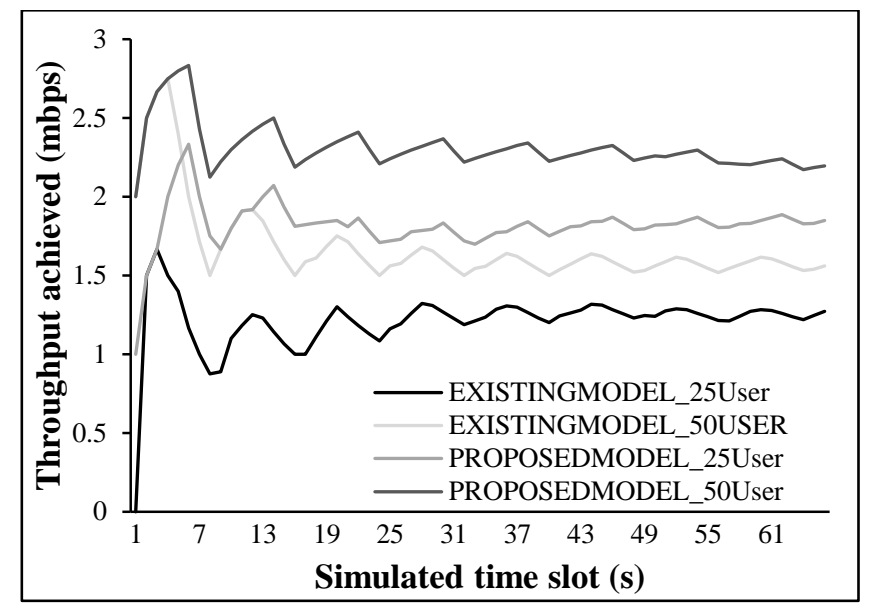

Fig.3. Throughput achieved per slot for varied sensor devices

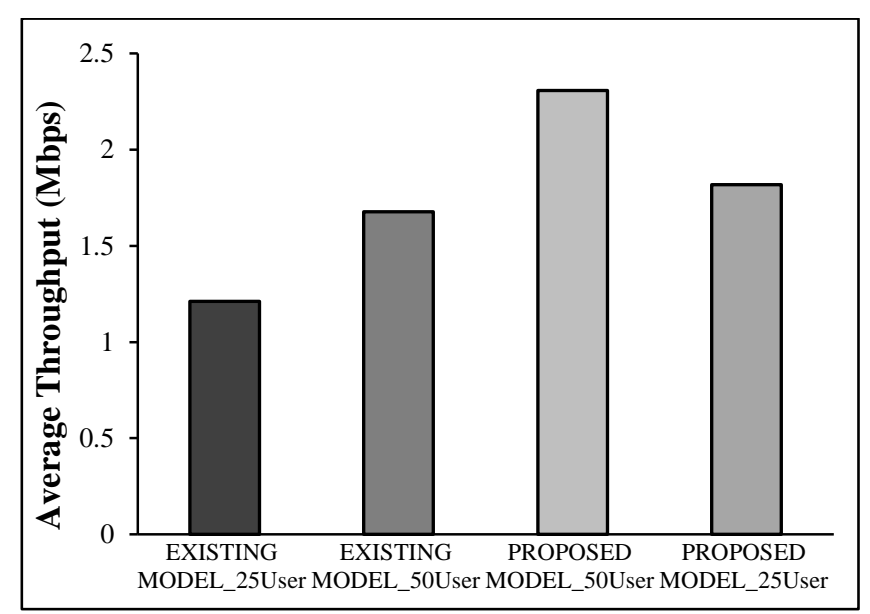

Fig.4. Average Throughput achieved per slot for varied sensor devices 
In Fig.5, the simulation outcome of number of packet transmitted successfully is shown. The sensor nodes are varied from 25 and 50 sensor devices and outcome shows that the proposed model improves packet transmission over existing model. A successful improvement of $27.39 \%$ is achieved when sensor node is equal to 25 and throughput improvement of $27.38 \%$ is achieved when sensor node is equal to 50 which are shown in Fig.5.

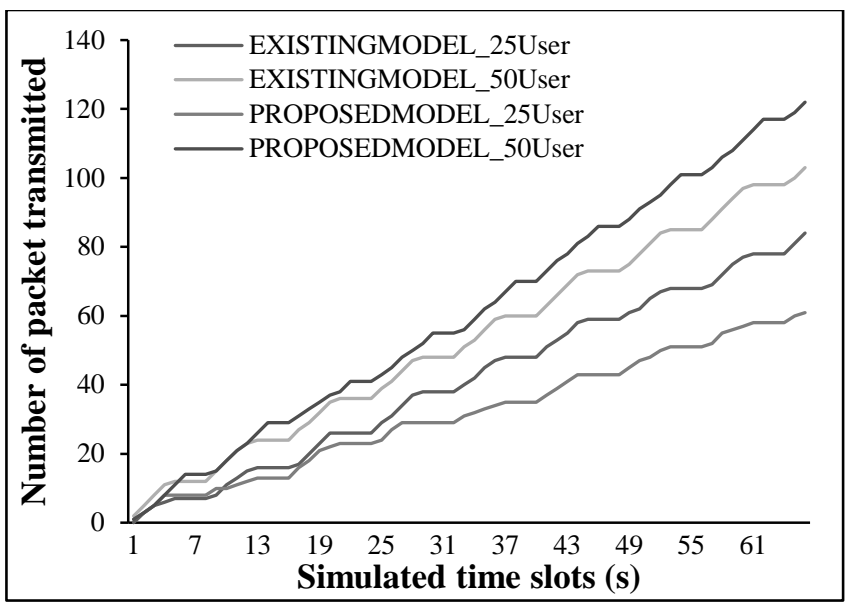

Fig.5. Successful packet transmission for varied sensor devices

To evaluate the performance of packet Collision and throughput performance of both proposed and existing approach the node size is fixed to 25 sensor devices and time slot is varied from 4, 6 and 8 . In Fig.6, the simulation outcome of throughput achieved for varied time slot is shown. The outcome shows that the proposed model improves throughput over existing model. A throughput improvement of $24.79 \%$ is achieved when time slot is equal to 4 , throughput improvement of $29.68 \%$ is achieved when time slot is equal to 6 and through improvement of $16.38 \%$ is achieved when time slot is equal to 8 . The Fig.6 shows when we increase the time slot the average throughput achieved also increases due to unutilized slots for both proposed and existing approach.

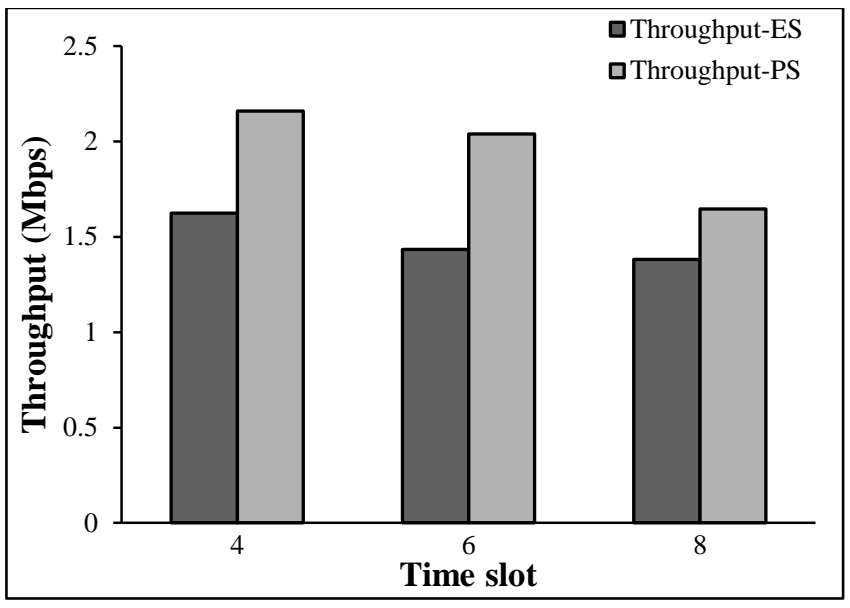

Fig.6. Average throughput performance for varied time slots

In Fig.7, the simulation outcome of collision achieved for varied time slot is shown. The outcome shows that the proposed model reduces collision over existing model. A collision reduction of $8.09 \%$ is achieved when time slot is equal to 4 , collision reduction of $28.68 \%$ is achieved when time slot is equal to 6 and collision reduction of $22.38 \%$ is achieved when time slot is equal to 8 . The Fig.6 shows when we increase the time slot the collision decreases due to slots available for both proposed and existing approach.

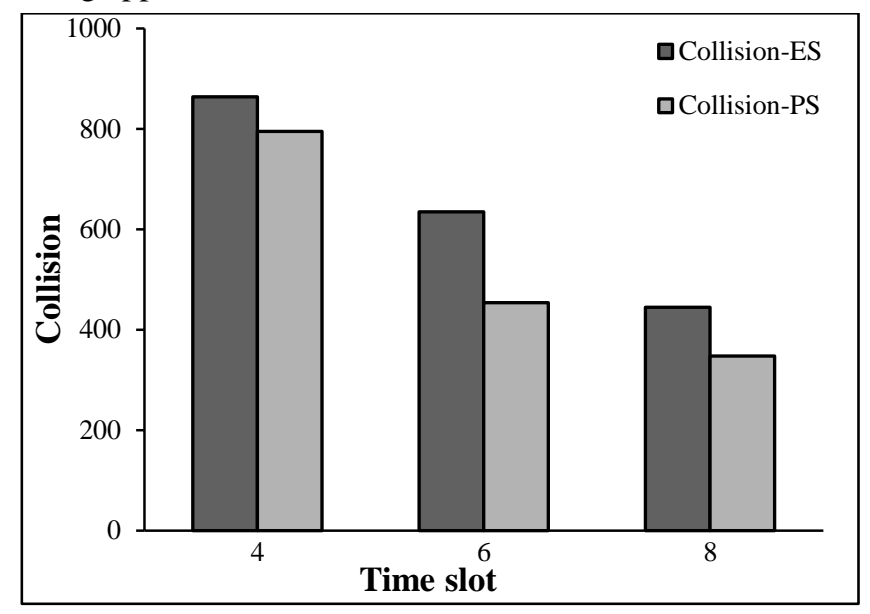

Fig.7. Collision performance for varied time slots

It is seen from the result obtained that proposed model is efficient in terms of throughput, successful packet transmission and reduced packet collision when compared to existing cognitive radio based wireless sensor network.

\section{CONCLUSION}

In cognitive radio network the spectrum is mostly not occupied by the primary licensed user all the time. Utilizing these unoccupied spectrums plays key role providing efficient service end user. The wireless sensor network adopts cognitive radio to transmit data using unoccupied channel. In this work, a cognitive networking with dynamic opportunistic spectrum access mechanism for WSNs is introduced. The channel availability of likelihood distribution is computed using continuous-time Markov chain considering primary transmitting users temporal channel usage channel pattern and spatial distribution. The experiment is conducted to evaluate the performance of proposed model in terms of collision, successful packet transmission and throughput efficiency by varying network density size. The outcome shows significant performance improvement. An improvement of $16.3 \%$ is achieved in terms of collision reduction, an improvement of $30.8 \%$ in terms of successful packet transmission is achieved and an improvement of $30.8 \%$ in terms of throughput is achieved for varied network density. An improvement of $19.3 \%$ is achieved in terms of collision reduction and an improvement of $23.8 \%$ in terms of throughput is achieved for varied time slots. The outcome of proposed model is scalable in terms of network density. The future model would consider different MAC and modulation schemes and would consider developing non-cooperative game theory model for efficient channel access.

\section{REFERENCES}

[1] I.F. Akyildiz, W. Su, Y. Sankarasubramaniam and E. Cayirci, "Wireless Sensor Networks: A Survey", Computer Networks, Vol. 38, No. 4, pp. 393-422, 2002.

[2] Mainetti Luca et al., "Evolution of wireless sensor networks towards the Internet of Things: A survey", Proceedings of 
IEEE International Conference on Software, Telecommunications and Computer Networks, pp. 15-17, 2011.

[3] Tao Hua et al., "Intelligent Photovoltaic Monitoring based on Solar Irradiance Big Data and Wireless Sensor Networks", Ad Hoc Networks, Vol. 35, No. 5, pp. 127-136, 2015.

[4] Bin He and Yonggang Li, "Big Data Reduction and Optimization in Sensor Monitoring Network", Journal of Applied Mathematics, Vol. 2014, pp. 1-8, 2014.

[5] Jiachen Yang, Yancong Lin and Zhihan Lv, "A SelfAssessment Stereo Capture Model Applicable to the Internet of Things", Sensors, Vol. 15, No. 8, pp. 20925-20944, 2015.

[6] Jiachen Yang, Shudong He, Yancong Lin and Zhihan Lv, "Multimedia Cloud Transmission and Storage System based on Internet of Things", Multimedia Tools and Applications, Vol. 76, No. 17, pp. 1-16, 2015.

[7] Elias Yaacoub et al., "Cooperative Wireless Sensor Networks for Green Internet of Things", Proceedings of $8^{\text {th }}$ ACM Symposium on QoS and Security for Wireless and Mobile Networks, pp. 79-80, 2012.

[8] Jiachen Yang, Shudong He, Yancong Lin and Zhihan Lv, "A Real-Time Monitoring System of Industry Carbon Monoxide based on Wireless Sensor Networks", Sensors, Vol. 15, No. 11, pp. 29535-29546, 2015.

[9] D. Jiang et al., "A Collaborative Multi-Hop Routing Algorithm for Maximum Achievable Rate", Journal of Network and Computer Applications, Vol. 57, pp. 182-191, 2015.

[10] H Zain Eldin, M.A. Elhosseini and H.A. Ali, "Image Compression Algorithms in Wireless Multimedia Sensor Networks: A Survey", Ain Shams Engineering Journal, Vol. 6, No. 2, pp. 481-490, 2015.

[11] Paola G. Vinueza Naranjo et al., "P-SEP: A Prolong Stable Election Routing Algorithm for Energy-Limited Heterogeneous Fog-Supported Wireless Sensor Networks", The Journal of Supercomputing, Vol. 73, No. 2, pp. 1-23, 2016.

[12] G. Zhou, J.A. Stankovic and S.H. Son, "Crowded Spectrum in Wireless Sensor Networks", Proceedings of $3^{\text {rd }}$ Workshop on Embedded Networked Sensors, pp. 1-5, 2006.

[13] J. Yang et al., "A Low-Power and Portable Biomedical Device for Respiratory Monitoring with a Stable Power Source", Sensors, Vol. 15, No. 8, pp. 19618-19632, 2015.

[14] S. Maghsudi and S. Stanczak, "Hybrid CentralizedDistributed Resource Allocation for Device-to-Device Communication Underlaying Cellular Networks", IEEE Transactions on Vehicular Technology, Vol. 65, No. 4, pp. 2481-2495, 2016.

[15] D. Jiang, Y. Wang and C. Yao, "An Effective Dynamic Spectrum Access Algorithm for Multi-Hop Cognitive Wireless Networks", Computer Networks, Vol. 84, No. 19, pp. 1-16, 2015.

[16] T.A. Myrvoll and J.E. Hakegard, "Dynamic Spectrum Access in Realistic Environments using Reinforcement Learning", Proceedings of International Symposium on Communications and Information Technologies, pp. 465470, 2012.

[17] Yuhua Xu, Jinlong Wang, Qihui Wu, A. Anpalagan and YuDong Yao. "Opportunistic Spectrum Access in Unknown
Dynamic Environment: A Game-Theoretic Stochastic Learning Solution", IEEE Transactions on Wireless Communications, Vol. 11, No. 4, pp. 1380-1391, 2012.

[18] S. Vakili, K. Liu and Q. Zhao, "Deterministic Sequencing of Exploration and Exploitation for Multi-Armed Bandit Problems", IEEE Journal of Selected Topics in Signal Processing, Vol. 59, No. 3, pp. 1902-1916, 2013.

[19] Y. Gai and B. Krishnamachari, "Decentralized Online Learning Algorithms for Opportunistic Spectrum Access", Proceedings of IEEE Global Communication Conference, pp. 1-6, 2011.

[20] A. Anandkumar, N. Michael, A. Tang and A. Swami, "Distributed Algorithms for Learning and Cognitive Medium Access with Logarithmic Regret", IEEE Journal on Selected Areas in Communications, Vol. 29, No. 4, pp. 731745, 2011.

[21] Marjan Zandi, Min Dong and Ali Grami, "Decentralized Spectrum Learning and Access Adaptive to Channel Availability Distribution in Primary Network", Proceedings of $14^{\text {th }}$ Workshop on Signal Processing Advances in Wireless Communications, pp. 130-134, 2013.

[22] M. Lelarge, A. Proutiere and M S. Talebi, "Spectrum Bandit Optimization", Proceedings of Information Theory Workshop, pp. 1-5, 2013.

[23] J. Zhu, Y. Song, D. Jiang and H. Song, "Multi-Armed Bandit Channel Access Scheme with Cognitive Radio Technology in Wireless Sensor Networks for the Internet of Things", IEEE Access, Vol. 4, pp. 4609-4617, 2016.

[24] Y. Xu, Q. Wu, J. Wang, L. Shen and A. Anpalagan, "Robust Multiuser Sequential Channel Sensing and Access in Dynamic Cognitive Radio Networks: Potential Games and Stochastic Learning", IEEE Transactions on Vehicular Technology, Vol. 64, No. 8, pp. 3594-3607, 2015.

[25] A. Jamal, C.K. Tham and W.C. Wong, "CR-WSN MAC: An Energy Efficient and Spectrum Aware MAC Protocol for Cognitive Radio Sensor Network", Proceedings of $9^{\text {th }}$ International Conference on Cognitive Radio Oriented Wireless Networks and Communications, pp. 67-72, 2014.

[26] M. Hefnawi, "Large-Scale Multi-Cluster MIMO Approach for Cognitive Radio Sensor Networks", IEEE Sensors Journal, Vol. 16, No. 11, pp. 4418-4424, 2016.

[27] A. Ahmad, S. Ahmad, M.H. Rehmani and N.U. Hassan, "A Survey on Radio Resource Allocation in Cognitive Radio Sensor Networks", IEEE Communications Surveys and Tutorials, Vol. 17, No. 2, pp. 888-917, 2015.

[28] Ibrahim Mustapha, Borhanuddin M. Ali, A. Sala, M.F.A. Rasid and H. Mohamad, "An Energy Efficient Reinforcement Learning based Cooperative Channel Sensing for Cognitive Radio Sensor Networks", Pervasive and Mobile Computing, Vol. 35, pp. 165-184, 2017.

[29] Yan Jiao and Inwhee Joe, "Markov Model-Based Energy Efficiency Spectrum Sensing in Cognitive Radio Sensor Networks", Journal of Computer Networks and Communications, Vol. 6, pp. 1-8, 2016.

[30] P. Spachos and D. Hatzinakos, "Real-Time Indoor Carbon Dioxide Monitoring Through Cognitive Wireless Sensor Networks", IEEE Sensors Journal, Vol. 16, No. 2, pp. 506$514,2016$. 